\title{
De l'affect de la loi au commandement de la Vie
}

Yukihiro HATTORI

Université de Kyoto

Le problème de l'action s'impose à Michel Henry dès son essai sur Maine de Biran, dans lequel il dénonce le concept traditionnel d'action, compris comme réalisation d'une intention objectivant ${ }^{1}$. Le problème est alors celui d'une déchirure ontologique entre l'intention et l'action, entre la volonté et son effectuation, ou encore entre la cause intelligible de l'action et son effet sensible. Pour dépasser cet abîme déjà aperçu par Kant, Henry cherche à forger à nouveaux frais le concept d'action. L'un des moments essentiels de cette tentative se présente dans son analyse de la conception kantienne du respect ${ }^{2}$ dans L'essence de la manifestation. Quelle est alors la signification de cet intérêt pour l'action dans le cadre de la phénoménologie de la vie ? L'objectif de cet article consiste à expliciter le sens de cette analyse au sein de la pensée de Henry, en examinant le développement de la critique du respect kantien dans l'analyse du «commandement de la Vie » dans C'est moi la vérité. Pour y parvenir, nous parcourrons d'abord l'analyse henryenne du respect pour la loi morale chez Kant, pour ensuite dégager la différence essentielle entre la loi morale et la Loi provenant de la Vie elle-même, à partir d'une opposition entre la loi vétéro-testamentaire et l'amour néo-testamentaire. Enfin, nous chercherons à élucider l'enjeu fondamental dont est porteuse cette dernière opposition.

\section{La critique du concept kantien de respect}

Afin de préciser la structure originaire de l'affectivité dans L'essence de la manifestation, Henry oppose cette dernière au concept kantien de respect. Dans cette critique de Kant, quel moment de l'analyse du respect s'agit-il toutefois d'attaquer ? Après avoir présenté l'importance du concept de respect dans les Fondements de la métaphysique des mours, Kant thématise ce concept primordial dans son projet de philosophie morale. Dans un chapitre de la Critique de la raison pratique, intitulé « Des ressorts de la raison pratique pure », il s'efforce de montrer comment la loi morale détermine la volonté humaine en la dirigeant vers une action. Si la moralité

\footnotetext{
${ }^{1}$ Cf. Michel Henry, PPC, p. 276-279.

${ }^{2}$ Le problème du respect chez Michel Henry a déjà été traité par John Llewelyn, dont l'analyse s’en tient pourtant à L'essence de la manifestation, en suggérant seulement son lien étroit avec C'est moi la vérité. Cf. John Llewelyn, "Le respect comme affectivité effective ", dans Michel Henry, L'épreuve de la vie, A. David et J. Greisch (éd.), Paris, Cerf, 2001, p. 453-465.
} 
nous demande de réaliser l'action non pas pour son objectif, mais plutôt pour son motif, et si l'action s'accomplit exclusivement pour s'accorder avec la loi morale, elle doit alors être une action motivée exclusivement par la loi morale ${ }^{3}$.

Mais comment la seule loi morale, suprasensible, est-elle capable de susciter une action tout en déterminant elle-même la volonté opérant dans le monde sensible ? À la différence de la volonté absolue de Dieu, l'action humaine, volontaire ou involontaire, ne se réalise qu'en étant motivée par le «mobile » ou le «ressort» qui vient déterminer cette action en dehors d'elle-même ${ }^{4}$. C'est une telle motivation qui rendrait le plus souvent possible l'action humaine, suscitée ou bien par l'affection sensible ou bien par la loi suprasensible. Quel que soit son ressort, l'agir de l'homme trouve son caractère propre dans cette déchirure, partagé entre le sensible et l'intelligible. La loi morale s'oppose en ce sens à toutes les inclinations sensibles. Cette exigence d'éliminer les obstacles à l'action morale par la raison pratique pure donne lieu à un sentiment désagréable, puisqu'elle impose une comparaison entre la loi et les inclinations. Pour «nous débarrasser d'un obstacle », elle nous pousse à lui « résister » et ainsi à nous « humilier » jusqu’à nous élever à la loi morale.

Ce sentiment éprouvé comme «combat» entre la loi morale et l'inclination constitue le sentiment du respect ${ }^{5}$. Aussitôt que la loi morale rejette l'inclination comme obstacle, ce combat ne permet pas seulement de détruire la présomption qui n'est que sensible et de susciter le sentiment de l' " humiliation », mais il conduit en outre à remonter à la loi pour respecter celle-ci en tenant à distance les inclinations. La loi morale a ainsi sur le sujet une "double action » : elle le conduit à résister aux inclinations et à remonter vers la loi morale.

Le respect se présente donc selon une dualité essentielle. L’affection du sujet par la loi morale ne peut manquer d'accompagner l'expérience sensible vécue comme humiliation, si bien que la loi morale est capable d'avoir un effet sur le sensible, pourtant à première vue hétérogène à elle-même. C'est précisément sur une telle dualité du respect que se concentre la lecture henryenne. Aux yeux de Henry, l'essentiel du respect est son caractère doublement affectif ${ }^{6}$. Le respect résulte de la loi morale, mais, en tant qu'effet de celle-ci, il suscite le sentiment sensible de l'humiliation. Sa cause est purement intellectuelle, mais son effet n'est que sensible. Ce que Henry nomme, dans sa critique du respect, un "paradoxe incompréhensible " ${ }^{7}$, c'est cette dualité elle-même, qui sépare la cause intellectuelle et l'effet sensible. Incompréhensible, puisque l'unité d'une telle dualité, qui creuse dans l'humanité de l'homme "sa division en deux éléments hétérogènes », la

\footnotetext{
${ }^{3}$ Cf. Emmanuel Kant, Critique de la raison pratique, trad. par J.-P. Fussler, Paris, GF Flammarion, 2003, p. 181.

${ }^{4}$ Ibid., p. 185.

${ }^{5}$ Ibid., p. 184.

${ }^{6}$ Michel Henry, EM, p. 653.

${ }^{7}$ Ibid., p. 652.
} 
sensibilité et la raison, reste foncièrement indéterminée chez Kant. Il s'ensuit qu'il faut fonder la "dimension d'unité à laquelle elles [la sensibilité et la raison] coappartiennent par essence ${ }^{8}$, sans quoi l'idée d'une affection pathologique de la loi morale, sur laquelle repose le respect comme «sentiment moral», ne serait qu' « une absurdité »" .

Kant voit assurément que le respect n'est pas seulement un sentiment sensible, mais aussi un sentiment singulier, dont la singularité réside dans son caractère non-sensible, à savoir intellectuel, en tant qu'il a son origine dans la raison pratique uniquement. Pourtant, au lieu de saisir le sens originaire du sentiment intellectuel propre au respect, Kant reconduit celui-ci à la structure de la représentation. Lorsque le sujet choisit librement la soumission non pas aux inclinations, mais à la loi morale, pour juger qu'elle est ce à quoi il doit se soumettre, la loi morale affecte le sujet, c'est-à-dire, pour Kant, qu'elle est représentée par lui : «La représentation de la loi, c'est là la condition ontologique du respect. » ${ }^{10}$ Autrement dit, le caractère intellectuel du respect repose sur son affection par la loi morale. D'ailleurs, si le respect fonctionne comme sentiment sensible de l'humiliation, c'est que, dans l'exclusion des inclinations, le respect peut affecter la nature psychophysique de l'homme pour le conduire à réaliser l'action sous la contrainte pratique, par sentiment du «devoir». Le caractère sensible de l'être-affecté du respect repose sur la sensibilité empirique, qui est l'affection de la forme par la matière qui lui est étrangère.

C'est ainsi que, selon Henry, le respect intellectuel et sensible "se présente nécessairement comme une détermination de l'affectivité ${ }^{11}$. La coappartenance de la sensibilité et de la raison à l'être-affecté du respect réside dans l'affectivité, dont la conceptualisation fait pourtant «totalement défaut à Kant " ${ }^{12}$. Si la condition du respect consiste dans la «structure ontologique de l'affection pure», pourtant «l'affectivité du respect est laissée de côté ${ }^{13}$. C'est la raison pour laquelle Henry reproche à Kant d'escamoter l'essence de l'affectivité, notamment dans sa tentative de concilier la double affection par la loi morale ${ }^{14}$.

Pourquoi l'affectivité fait-elle défaut chez Kant? Si l'être-affecté signifie l'être-motivé, le respect permet le retournement des ressorts sensibles de l'« action »

\footnotetext{
${ }^{8}$ Ibid., p. 660.

${ }^{9}$ Ibid., p. 659. C'est grâce à cette affection mystérieuse que la loi morale peut susciter le sentiment, et en tant que telle, elle peut être le fondement de la détermination subjective comme « ressort pour l'action ». Kant se limite cependant à dire que «l'on trouve insondable pour la raison spéculative cette influence d'une idée purement intellectuelle sur le sentiment » (Emmanuel Kant, Critique de la raison pratique, op. cit., p. 190, c'est moi qui souligne). Cette liaison «insondable» entre la sensibilité et la raison, Henry la cherche dans l'affectivité.

${ }^{10}$ Ibid., p. 656.

${ }^{11}$ Ibid., p. 661.

${ }^{12}$ Ibid., p. 656

${ }^{13}$ Ibid., p. 665

${ }^{14}$ Cf. Ibid., p. 654.
} 
en ressorts intellectuels, un passage de la causalité physique à la causalité morale. Cette causalité morale, ou "moralité », qui rend possible l'action en tant qu'elle ressort de la «liberté », repose sur une relation pure entre le sujet et la loi morale. Mais, pour la volonté, la loi morale ne peut jamais se donner empiriquement comme fait de l'intuition ; elle se donne plutôt à nous comme " originairement législative ", comme « fondement de la détermination ». Elle est l'« objet de la volonté » qui nous conditionne. Certes, Kant affirme que l'on peut être «conscient» de la loi morale comme " exigence pratique ", " fait de la raison pure », mais il ne cherche pas à se demander comment on peut le devenir ${ }^{15}$.

C'est dans ce sens seulement que la loi morale est dite « transcendante ». Aux yeux de Henry, Kant laisse le sujet déchiré entre le monde sensible et la loi morale, car pour lui l'homme en tant que créature «ne peut jamais être entièrement libre de désirs et d'inclinations $»^{16}$. L'homme fini ne peut être qu' « hétérogène ${ }^{17}$ à la réalité qui est sa personnalité intelligible. C'est pourquoi l'affectivité du respect, qui contient la déchirure entre la cause et l'effet du respect, reste encore obscure.

Or, Henry rappelle que Kant ne cesse de supposer qu'une affectivité au sens propre se trouve dans "l'amour", certes "de façon inaperçue " ${ }^{18}$, et ce même si Kant reproche à celui-ci sa nature pathologique. À la différence du respect, aux yeux de Henry, c'est l'amour qui ne contient rien de transcendant, étranger qu'il est à l'affection sensible. Henry écrit : «L'amour signifie une détermination de l'action à partir de la structure interne de l'essence comprise dans son immanence radicale et dans ce qu'elle est originairement pour elle-même, comme auto-affection et comme affectivité. " ${ }^{19}$ Cette interprétation de l'amour comme auto-affection, qu'il a déjà livrée à propos du concept fichtéen d'amour ${ }^{20}$, fait ressortir que l'affectivité de l'amour réside dans l'auto-affection, qui est « l'auto-affection de l'acte qui pose la loi, dans l'affectivité de la raison elle-même en tant que pratique ${ }^{21}$. L'auto-affection de la volonté législative permet seulement que «le fait de la raison soit tiré de l'incertitude foncière " ${ }^{22}$. Dès lors, l'affectivité est nommée aussi « raison », en tant que fondement se révélant lui-même tel qu'il est ${ }^{23}$.

\footnotetext{
${ }^{15}$ Emmanuel Kant, Critique de la raison pratique, op. cit., p. 147.

${ }^{16}$ Ibid., p. 195.

${ }^{17}$ Michel Henry, EM, p. 663.

${ }^{18}$ Ibid., p. 665.

${ }^{19}$ Ibid.

${ }^{20}$ La notion d'amour est elle-même déjà analysée dans Michel Henry, BS, p. 113-114. Cf. aussi Michel Henry, EM, p. 378.

${ }^{21}$ Ibid., p. 666

${ }^{22}$ Ibid.

${ }^{23}$ Cf. Ibid., p. 109, où Henry écrit : « La Raison, enfin, est un sentiment ». Cf. aussi Michel Henry, EM, p. 668.
} 


\section{La consubstantialité immédiate de l'agir et du commandement de la Vie}

Henry affirme que l'essence de l'affectivité se trouve dans le christianisme, qui substitue l'amour à la Loi. Le christianisme ne repose plus sur la moralité de la loi, qui suppose la séparation de ce qui commande et de ce qui obéit, mais il découvre une « nouvelle détermination de l'existence effective et, par suite, de l'action elle-même comme modalité de cette existence " ${ }^{24}$. L'action ne se réalise pas dans le monde sensible, mais plutôt dans la « réalité affective spécifique ». Toutefois, dans L'essence de la manifestation, Henry ne déploie pas encore cette idée selon laquelle l'action ne viendrait pas de la loi transcendante, mais qu'elle serait la modalité de l'existence même. C'est dans C'est moi la vérité que, en reprenant la critique formulée à l'encontre de la loi - et cette fois, nommément, à l'égard de la Loi de l'Ancien Testament - Henry en vient à préciser le sens originaire de l'action comme modalité de l'existence affective, ou encore comme modalité de la vie.

Commentant les épîtres pauliniennes, Henry fournit une interprétation remarquable de la critique paulinienne de la Loi. Dans le régime vétéro-téstamentaire, l'action, soumise à la Loi, s'accomplit au gré d'un procès d’objectivation. L'action vétéro-tétamentaire consiste en un passage de ce qui ne se voit pas à ce qui se voit, selon une entrée dans la visibilité, un faire-voir-au-dehors, qui se dit aussi « produire » (pro-ducere). La Loi suppose en effet la distance ou l'extériorité entre ce qui commande et ce qui obéit en séparant le commandement de sa réalisation, et en réduisant cette dernière à la structure de la représentation. La Loi manque ainsi le pouvoir de faire naître l'action en l'homme. Provenant de la Loi qui est transcendante à l'égard du sujet agissant, l'action est pénétrée par le mode ekstatique de l'apparaître. Henry peut donc écrire : « la conformité de l'agir à la Loi, c’est la conformité d'un comportement objectif à un modèle idéal, au modèle de la Loi précisément — ce comportement objectif qui n’offre que l’apparence extérieure de l’agir dans la vérité du monde. ${ }^{25}$

À cette définition de l'action comme production, dont l'essentiel a déjà été présenté dans les ouvrages précédents tel que le $\mathrm{Marx}^{26}$, Henry oppose une autre, qui conçoit l'action comme processus invisible hors de la lumière de l'extériorité. Henry s'efforce alors de substituer au procès d'objectivation un procès de "génération " ${ }^{27}$. Si la loi vétéro-testamentaire implique que l'homme réalise l'action en la posant hors d'elle-même, le commandement de la vie néo-testamentaire met en œuvre l'action dans un s'éprouver soi-même. L'agir invisible est la réalisation immédiate et interne

\footnotetext{
${ }^{24}$ Ibid., p. 666.

${ }^{25}$ Michel Henry, CM, p. 226.

${ }^{26}$ Michel Henry, M I, p. 329.

${ }^{27}$ Michel Henry, CM, p. 228.
} 
de l'action au sein de la vie, qui n'est rien d'autre que la génération des vivants au sein de la Vie absolue, l’auto-génération de la vie.

La différence entre la réalisation « médiate » de l'action par la production (modèle vétéro-testamentaire) et celle, "immédiate ", de l'action par l'auto-génération (modèle néo-testamentaire), éclate également dans le contraste entre les deux types de rapport que l'action entretient avec sa condition. D'un côté, ce rapport de l'action au commandement s'explique comme une conformité extérieure et idéale de l'action à la Loi, qui pose celle-là hors d'elle-même par un procès de la production. De l'autre, dans le cas de l'auto-génération, le commandement est identique à l'agir ; «le commandement et l'agir vont ensemble ${ }^{28}$. Si la critique paulinienne de la Loi renvoie à la figure du Christ en rejetant l'obéissance à la Loi, c'est que le Christ n'est rien d'autre que la figure concrète qui exprime la consubstantialité de l'agir et de sa condition qu'est la vie elle-même au sein du procès de l'auto-génération. C'est ainsi que le sens de la critique paulinienne de la Loi ne consiste plus dans la substitution de la croyance, ou de la confession du péché, à l’observation de la Loi, mais dans celle de la génération immédiate de l'agir à la détermination médiate et relative de l'agir par la Loi transcendante.

S'il en est ainsi, ce contraste entre deux types de rapport de l'action au commandement suggère, nous semble-t-il, un parallélisme avec le modèle kantien de la loi morale présenté précédemment. En effet, de même que le respect kantien pour la loi morale suppose une distance entre la loi morale et le sujet agissant, la loi vétéro-testamentaire requiert elle aussi le même type de distance entre ce qui commande et ce qui obéit. De même que l'affectivité du respect s'accomplit elle-même dans l'auto-affection de la volonté législative, l'auto-génération de la vie implique l'immédiateté entre ce qui commande et ce qui obéit. L'analyse de l'action menée par Henry en 1996 en suivant les deux mondes bibliques ou judéo-chrétiens poursuit donc le même chemin que celui emprunté par la critique du respect kantien en $1963^{29}$.

\section{L’amour comme seule action possible dans la liberté du vivre}

Que signifie alors la différence entre le rapport médiat de la Loi à l'action visible dans un monde vétéro-testamentaire et le rapport immédiat du commandement de la vie à l'agir invisible ? Revenons brièvement sur l'analyse kantienne du respect. Le respect n'est pas seulement affecté — soit par la loi morale, soit par les inclinations mais il s'accompagne aussi de l'acte intellectuel du «jugement de la raison», mobilisé pour comparer loi morale et inclinations. Or, l’homme fini, qui se lie inévitablement à l'affection sensible, est obligé de faire un choix entre le sensible et la

${ }^{28}$ Ibid., p. 236.

${ }^{29}$ Henry revient aussi sur l'analyse kantienne du respect en 1996. Cf. Michel Henry, CM, p. 235. 
loi morale. En ce sens, Kant n'hésite pas à affirmer que la soumission de l'homme à la loi morale n'est rien d'autre que libre. C'est en raison de ce choix ouvert que l'homme se trouve dans la double possibilité : l'une, d'obéissance à la loi morale, et l'autre, de désobéissance.

En revanche, Henry vise à éliminer d'une façon résolue cette possibilité de désobéissance, en refusant l'intervention de toute affection sensible extérieure. Afin de substituer le « Fils » à l' " homme », il fait porter l'accent sur la « pré-destination », inscrite dans l'agir du Fils, qui « a déjà accompli sa prescription». En effet, le commandement qui ordonne cette prescription «s'est déjà accompli » en chaque Fils $^{30}$. Henry affirme donc :

Quand l'« homme » est compris dans sa condition de Fils [...], une donne préalable existe, là aussi. Cette donne n'est plus une programmation, mais une pré-destination [...] en vertu de laquelle [...] l'homme est destiné à être ce vivant [...], ne pouvant accomplir sa propre essence que dans l'essence de cette Vie absolue. $^{31}$

Dans la prédestination, idée déjà anticipée dans $L$ 'essence de la manifestation ${ }^{32}$, il n'y aurait aucune liberté, et sans elle les notions d' " éthique » et aussi de « commandement » n'auraient pas de sens. Cependant, à la différence de L'essence de la manifestation, qui ne cesse de reconduire la liberté humaine à la transcendance, Henry déclare, dans C'est moi la vérité, qu’il existe une liberté dans la vie. Une fois que le commandement de la Vie génère l'ego, il « contient la liberté requise par tout commandement éthique $»^{33}$. Henry reprend le thème de la liberté pour le reformuler à la lumière de sa théorie du commandement de la Vie. Il s'agit là, en l'occurrence, de redéfinir la liberté comme « liberté de vivre » ${ }^{34}$, qui n'est plus l'effectuation objective de ma volonté dans son indépendance à l'égard de la causalité sensible, mais qui désigne « la mise en œuvre effective de chacun de mes pouvoirs » ${ }^{35}$, en tant que la vie s'accomplit comme « Je peux $»^{36}$.

Comment comprendre un tel élargissement du sens de la liberté ? Si le Fils est libre d'agir, il se trouve dans la possession parfaite de son «pouvoir », mais ceci a pour condition qu'il ne tienne son pouvoir que de lui-même. Sans tomber dans l'« illusion transcendantale ", qui consiste à prendre à tort son propre fond pour soi-même, le Fils accomplit son agir dans un rapport immédiat au « Père ». La liberté de l’action au sens

${ }^{30}$ Ibid., p. 231

${ }^{31}$ Ibid., p. 233.

${ }^{32}$ Cf. Michel Henry, EM, p. 816 et 824.

${ }^{33}$ Michel Henry, CM, p. 231.

${ }^{34}$ Ibid., p. 230.

${ }^{35}$ Ibid., p. 178.

${ }^{36}$ Henry substitue ainsi la liberté du pouvoir à la liberté du vouloir, qui suppose « l'extériorité de ce qu'il veut » (Michel Henry, EM, p. 817), en vue d'établir l'action réalisable avant sa rencontre avec le monde sensible, tandis qu'il reconduit dans sa lecture de Schopenhauer la relation de la volonté à elle-même à l'auto-affection. Cf. Michel Henry, GP, p. 208. 
henryen consiste non pas dans l'effectuation externe et, en quelque sorte, facultative de la Loi dans le monde sensible, mais plutôt dans la réalisation interne et irrécusable du commandement de la Vie dans la vie elle-même.

Le sens de l'action libre subit ainsi une mutation décisive. Henry insiste sur la continuité sans rupture du procès de l'auto-génération de la vie. Il déclare : « La vie ne connaît pas le repos du dimanche ou du samedi — ce qui vaut mieux d'ailleurs pour l'ensemble des vivants. ${ }^{37}$ Cela revient à dire qu'il n'y a aucune possibilité de rejet de la prescription, puisque cette donation de la vie aux vivants précède absolument toute décision ${ }^{38}$. Dès lors, le commandement de la Vie et la liberté sont bien compatibles, dans la mesure où celle-ci signifie à la fois l'accomplissement de l'action et l'obéissance au commandement. L'accomplissement de l'action libre ne suppose aucune séparation kantienne de la cause intellectuelle et de l'effet sensible, car la cause de l'action et son effet appartiennent, pour Henry, au seul et même domaine de la vie. C'est ainsi que l'action libre signifie, non pas réaliser dans le monde sensible la volonté fondée sur la loi morale, mais faire baigner hors du monde le « Je peux » engendré au sein de la génération de la vie.

Or, qu'est-ce que commande le commandement de la Vie comme action? Aimer. Après son interprétation de l'amour dans L'essence de la manifestation, Henry réintroduit celle-ci pour achever la théorie du commandement de la vie et aussi pour compléter sa critique de la moralité kantienne : « ce que Kant n’aperçoit pas, c’est que dans le christianisme le commandement d'amour n'est pas une loi éthique». L'homme ne peut en effet se persuader d'aimer, mais, en deçà de toute décision, il « porte en lui cet amour comme ce qui l'engendre à chaque instant » ${ }^{39}$. L'essence de l'amour comme auto-affection consiste à s'aimer soi-même dans l'étreinte pathétique de la vie. Le commandement de la vie est donc identique au «commandement d'amour ». Par conséquent, on en vient ici à la formulation achevée du sens de l'amour comme auto-affection. La vie commande l'amour, puisque l'amour est la seule action possible qui se réalise, non pas dans le monde sensible, mais au sein de la vie elle-même. C’est précisément comme amour que s'explique la réalisation interne de l'action soumise au commandement de la Vie.

\footnotetext{
${ }^{37}$ Michel Henry, CM, p. 228.

${ }^{38}$ Cf. Michel Henry, «Quatre principes de phénoménologie », dans PV-I, où Henry montre le caractère irrécusable du commandement de la Vie en parlant de l'« appel de la vie » qui s'oppose à l'« appel de l'être » : «Et cette impossibilité de tout repli et de toute réplique, cette façon d'être acculé à soi, ajusté point par point à tout ce qu'on est, c'est l'épreuve éternelle et irrémissible, inlassable et sereine que la Vie fait de soi à tout instant, c'est la blessure qu'elle creuse en nous, qui est notre subjectivité même et ce qui fait de nous des vivants » (Ibid., p. 103).

${ }^{39}$ Michel Henry, CM, p. 235.
} 


\section{L’accomplissement de l'action dans la « réalité invisible "}

De ce qui précède, comment saisir l'enjeu fondamental propre à la conception henryenne de ce commandement inéluctable et de la prédestination irrécusable du Fils ? Il convient à présent de rappeler que c'est dans la prédestination irrécusable que Henry s'efforce de situer le sens insigne du salut chrétien. Indépendamment de l'interprétation proprement théologique du salut, il conçoit la remontée à la vie absolue comme économie du salut, lequel se présente finalement comme « béatitude ». Si le salut consiste dans la béatitude, qui vient de la souffrance et de la jouissance constitutives de la structure antinomique de la vie, et si, en tant qu' « accomplissement » ${ }^{40}$, la béatitude n'est autre que l'objectif ou la fin ultime de ce mouvement originaire de la vie, cela revient alors à dire que, atteindre la fin de la vie, c'est identiquement atteindre le commencement de la vie.

Ce n'est pas le cas pour Kant, qui maintient quant à lui une séparation abyssale entre ces deux pôles extrêmes. Indépendamment de la question de savoir s'il y a une expérience privilégiée qui permet à l’homme, dépourvu de la sainteté de la volonté, de prendre immédiatement connaissance de la loi morale, Kant ne considère jamais le savoir immédiat de la loi morale comme étant l’objectif ultime de l'homme. Ce n'est pas en effet l'épreuve de la loi morale, mais le «souverain Bien », qui constitue cet objectif ultime et pourtant inatteignable pour l'homme fini, se divisant en dualité du sensible et de l'intelligible. Ce souverain Bien se compose, soulignons-le, non seulement de la vertu, mais aussi du « bonheur » comme de son « second élément » ${ }^{41}$. Cela implique que, pour l’homme fini, la « conséquence » pratique du souverain Bien ne se réalise que dans le monde sensible : « les actions qui visent à rendre effectif le souverain Bien appartiennent au monde sensible. ${ }^{42}$

En se limitant dans le «progrès allant à l'infini [Unendliche gehenden Progressus] ${ }^{43}$ pour réaliser le souverain Bien, Kant n’en vient jamais à unifier ou encore à « concilier » les deux rives de cet abîme fondamental. L'effort de Henry consiste plutôt à dépasser cet abîme, en se référant à la substitution radicale de l'amour néo-téstamentaire à la Loi vétéro-téstamentaire. Cette tentative est, nous semble-t-il, toute proche de la démarche du jeune Hegel, pour qui l'amour comme « pur sentiment de la vie » ${ }^{44}$ s’accomplit dans l’unité immédiate du Père et du Fils. Si, pour le jeune Hegel, la moralité kantienne, à la lumière de laquelle s’interprète l'esprit des Juifs, suppose l'unité du « concept », qui sépare la loi et ce qui y obéit, la religion de Jésus nous ramène alors à l'unité de l'amour qui concilie cette distance, dans la

\footnotetext{
${ }^{40}$ Ibid., p. 254.

${ }^{41}$ Emmanuel Kant, Critique de la raison pratique, op. cit., p. 242.

${ }^{42}$ Ibid., p. 243.

${ }^{43}$ Ibid., p. 246.

${ }^{44}$ Georg Wilhelm Friedrich Hegel, L'esprit du christianisme et son destin, trad. par J. Martin, Paris, Vrin, 1948, p. 77.
} 
mesure où « le rapport d'un fils à son père n'est pas un concept, [...] mais [le] rapport vivant d'êtres vivants, homogénéités de la vie » ${ }^{45}$.

C'est ainsi que le jeune Hegel en s'opposant à Kant parvient à l'unité vivante de ce qui commande et de ce qui obéit, tout comme c'est le cas dans la théorie henryenne du commandement de la vie. Pourtant, à la différence de l'amour henryen, l'amour hégelien ne se limite pas à l'unité vivante du fils et du père, mais, pour que cet amour devienne « religieux » et ainsi « universel » dans la « communauté chrétienne », il doit s'élever jusqu'à «se fondre avec le représenté, avec l'universel » ${ }^{46}$. Car l'unité vivante de l'amour ne recouvre pas encore la réalité objective. L'amour, en tant qu' «amour de tous pour tous » ${ }^{47}$, doit aller jusqu'à l'«intrusion d'un élément historique réel » dans la vie. Loin d'échapper à la réalité objective ou encore au «destin », l'amour se trouve saisi par lui.

L'important est que, tant pour Kant que pour Hegel, la réalité extérieure à la vie est la réalité irrécusable. C'est à ce point que se confronte l'effort henryen. La béatitude s'accomplit d'emblée dans le souffrir, qui consiste à s'éprouver soi-même sans jamais affronter une quelconque extériorité, qu'elle soit empirique ou intellectuelle. Avant même la rencontre avec le monde sensible ou objectif, l'amour comme auto-affection accomplit toujours déjà la communauté invisible des vivants, qui entendent toujours et déjà le bruit de la vie comme "Avant-absolu » ${ }^{48}$. Dans sa critique dirigée contre le jeune Hegel, qui prétend trouver dans le christianisme la distinction de la réalité visible et du Ciel invisible, Henry soutient fortement que la réalité n’est pas visible, mais qu'elle n'est qu'invisible. En s'opposant au jeune Hegel, il écrit : «Dans le christianisme rien ne s'oppose à la réalité, il n’y a rien d'autre que la vie. » ${ }^{49}$

Il est vrai que Henry n’en ignore jamais pour autant la réalité du monde visible dans le christianisme. Il écrit en effet : « loin de fuir la réalité de ce monde, le christianisme qui ne connaît que la vie n'a du même coup affaire qu'à cette réalité. ${ }^{50}$ Par la « réalité du monde », Henry entend néanmoins la condition de l'apparaître du monde. L'agir ne se réalise que dans cette condition invisible, qui n'a rien à voir avec la visibilité du monde lui-même : «ce qui apparaît dans le monde ne doit rien à

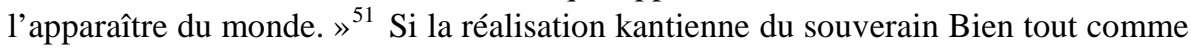
l'établissement hégélien de la communauté chrétienne ne va pas sans réalité visible, le concept henryen d'action confirme la transformation décisive du sens de la réalité du monde. Alors, « Le renversement radical des concepts relatifs à la réalité opéré par le christianisme ne va-t-il pas jusqu'à se retourner contre lui-même ? ${ }^{52}$. En considérant

${ }^{45}$ Ibid., p. 84.

${ }^{46}$ Ibid., p. 113.

${ }^{47}$ Ibid., p. 117-118.

${ }^{48}$ Michel Henry, CM, p. 199.

${ }^{49}$ Ibid., p. 297.

${ }^{50}$ Ibid., p. 306.

${ }^{51}$ Ibid., p. 304

52 Ibid., p. 302. 
l'apparaître du monde comme «illusoire », « idéal » ou « abstrait », Henry répond : « toute réalité se tient dans la vie invisible. ${ }^{53}$

\section{Conclusion}

Comme l'avait déjà suggéré son traité sur le Bonheur de Spinoza, qui recherchait la possibilité du salut dès la terre et sur terre, Henry ne cesse de se placer au sein de la tension entre réalité visible et réalité invisible, tension dont la conciliation est à jamais inatteignable pour l'homme fini. Par le passage de l'affect de la loi au commandement de la vie, Henry rend compte de la réalisation interne de l'action, qui n'est qu'amour, au sein de la vie elle-même, et par là il parvient à montrer la réalisation de la béatitude sur terre. Il nous semble que son argument quant au commandement de la Vie constitue une forme achevée de sa tentative générale de dépasser la tension entre des réalités antagonistes. Cette tentative ne va pourtant pas sans modifier le sens de la « terre ». La remontée de l'affect de la loi morale au commandement de la Vie, cette ascension vers la Vie absolue, doit rendre possible la descente vers le monde visible hétérogène à la vie elle-même, et ainsi l'itinéraire henryen nous ramène à une interrogation sur la genèse de la visibilité elle-même. Il nous ouvre à la question de savoir comment la réalité invisible accomplit son « union » avec la réalité visible, et également comment la vie se retrouve elle-même en dernier ressort comme la vie de tous les vivants et aussi de tous les hommes, bref comme « réalité vivante [lebendige Wesen] $»^{54}$.

\footnotetext{
${ }^{53}$ Ibid.

${ }^{54}$ Ibid., p. 116. Après la mort de Jésus, ses disciples se retrouvaient « rejetés dans la scission entre le visible et l'invisible » (Ibid., p. 115), où l'un manque l'autre ; et pourtant, dans le Ressuscité, « dans cette nouvelle union de l'esprit et du corps, l'opposition du vivant et du mort disparaissait, elle trouvait sa conciliation en un Dieu » (Ibid., p. 116).
} 
